EPJ Web of Conferences 52, 10005 (2013)

DOI: $10.1051 /$ epjconf/20135210005

(C) Owned by the authors, published by EDP Sciences, 2013

\title{
Very high energy gamma-ray emission of NGC 1275 and 3c382 and the possible activity nature of the Seyfert Galaxies
}

\author{
V.G. Sinitsyna ${ }^{1, a}$ and V.Y. Sinitsyna ${ }^{1}$ \\ ${ }^{1}$ P. N. Lebedev Physical Institute, Russian Academy of Science
}

\begin{abstract}
Galaxy NGC 1275 is the central dominant galaxy of the Perseus Cluster of Galaxies and is of Seyfert galaxy class. NGC 1275 is known as powerful X-ray and radio source. In 1996 year a new metagalactic source was detected by SHALON at TeV energies. This object was identified with Seyfert galaxy NGC1275. The image and spectral energy distribution of NGC 1275 as well as 3c382 are presented. The recent detection by the Fermi LAT of gamma-rays from the NGC1275 makes the observation of the energy E $>100 \mathrm{GeV}$ part of its broadband spectrum particularly interesting. The available high and very high energy data for NGC 1275 are well fitted in this model with three components.
\end{abstract}

\section{Introduction}

In the last years, high and very high energy $\gamma$-rays have come to play an important role in the study of Active Galactic Nuclei (AGNi). A big number of AGNi have been detected through the $\mathrm{MeV}$ to $\mathrm{TeV}$ energies. Also, active galaxies are intensely studied in all wavelengths. AGNi have been variously classified, but there are several types of active galaxies: Seyferts, quasars and blazars. It is (generally) supposed that the accreting supermassive black hole lies at the center of the active galaxy and the nucleus can produce a relativistic outflow of material. The search and investigation of $\gamma$-rays from radio galaxies is important for the understanding of the dynamics and structure of jets in AGNi. On the other hand, the problem in understanding of observed features, morphology of AGNi and jet dynamics is the unification of different types of active galaxies. The investigation of high energy $\gamma$-rays from radio-loud galaxies is important since it exhibits both the Seyfert-like and blazer-like radiated features in their wavelength spectra, which are disk related and jet-related respectively.

The SHALON observations of two type of radio galaxies - Seyfert galaxy NGC 1275[1-5] and Broad Line Radio Galaxy 3c 382 are presented. The observation results can help both to solve the problem of origin and acceleration of very high energy $\gamma$-rays thus the dynamics of outflows in the active galaxy and clarify the difference between the different classes of AGNi.

\section{$23 c 382$}

3c 382 is a typical Broad Line Radio Galaxy (BLRG), essentially identical to a quasar except that the optical lu-

\footnotetext{
a e-mail: sinits@sci.lebedev.ru
}

minosity. 3C382 is a well-known X-ray source. Its radio structure shows several quasar-like features. The core

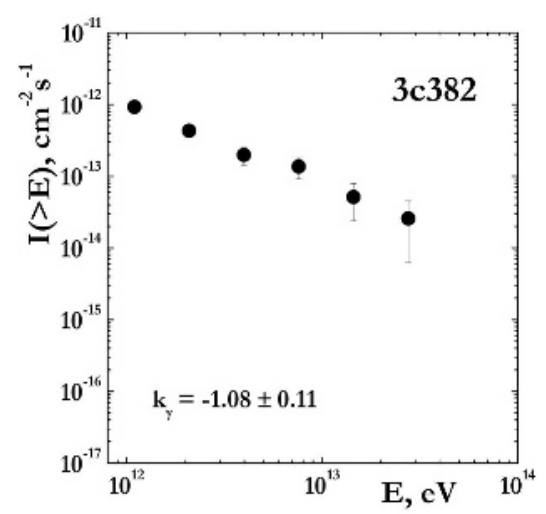

Figure 1. The gamma-quantum integral spectrum of $3 \mathrm{c} 382$ by SHALON.

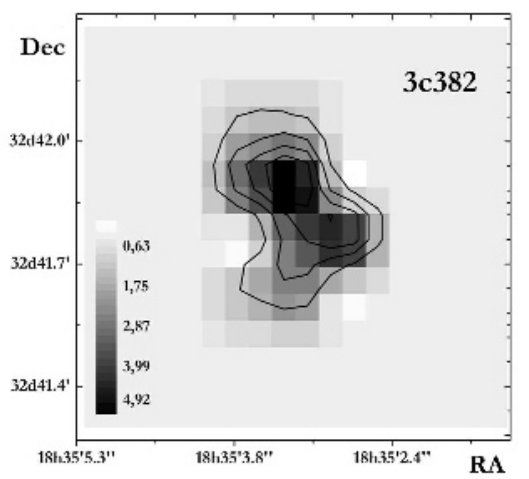

Figure 2. The 3c 382 image at energy range of $>800 \mathrm{GeV}$ by SHALON. 


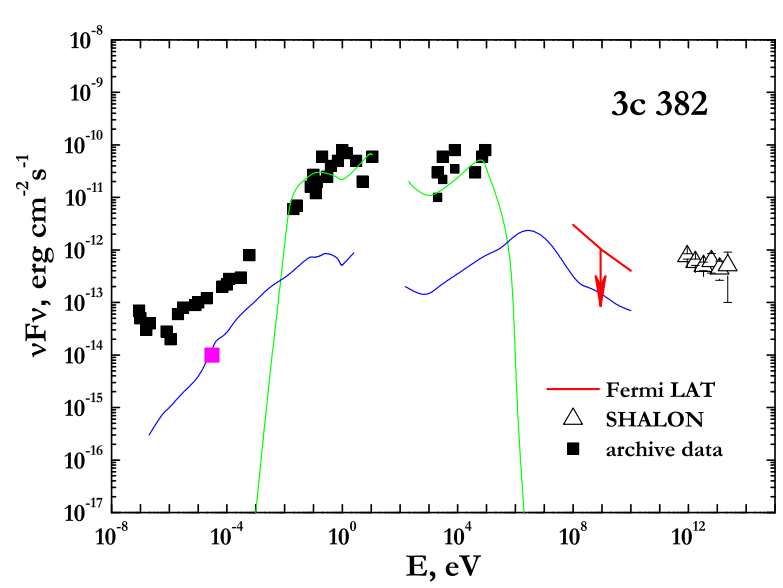

Figure 3. The spectral energy distribution of 3c 382 by SHALON (open triangles) in comparison with other experiments [6].

is very bright and there is a narrow jet with the compact hotspot and with the lobe opposite the jet. In contrast to blazars, the jets in BLRGs are not pointing directly toward the observer, and the relativistic beaming effects and the related jet dominance are only moderate, so it became possible to investigate both the accretion disk and the jet. In our observations we are mainly aimed on the searches for $\mathrm{TeV} \gamma$-ray emission form 3c 382 and the comparison with estimations [6].

3 c 382 has been detected by SHALON at TeV energies (in observations of 2009 - 2011 years) (Figs. 1, 2) with a statistical significance [7] of $6.7 \sigma$. The integral $\gamma$ ray flux above $0.8 \mathrm{TeV}$ was estimated as $(0.95 \pm 0.20) \times$ $10^{-12} \mathrm{~cm}^{-2} \mathrm{~s}^{-1}$. The energy spectrum of $\gamma$-rays in the observed energy region from $800 \mathrm{GeV}$ is described by the power law with the index $-1.08 \pm 0.11$ (Fig. 1). The observations of 3 c 382 at the high energies with Fermi LAT [6] have gave only the upper limit in the range $0.1-100$ GeV (Fig. 3).

\section{NGC 1275}

Galaxy clusters have been consider as sources of $\mathrm{TeV} \gamma$ rays emitted by high-energy protons and electrons accelerated by large scale structure formation shocks, galactic winds, or active galactic nuclei. The Perseus cluster of galaxies is one of the best studied clusters due to its proximity and its brightness. Galaxy NGC 1275 is the central dominant galaxy of the Perseus Cluster of Galaxies and is of Seyfert galaxy class. NGC1275 is known as powerful X-ray and radio source. The radio emission extends on larger scales, and shows an interaction with the cluster gas. Many studies explored correlations of X-ray, radio (Fig. 4), optical and ultraviolet emission [8].

In 1996 year a new metagalactic source are detected by SHALON at TeV energies (Figs. 4, 5, 6). This object was identified with Seyfert galaxy NGC 1275 [1-5]

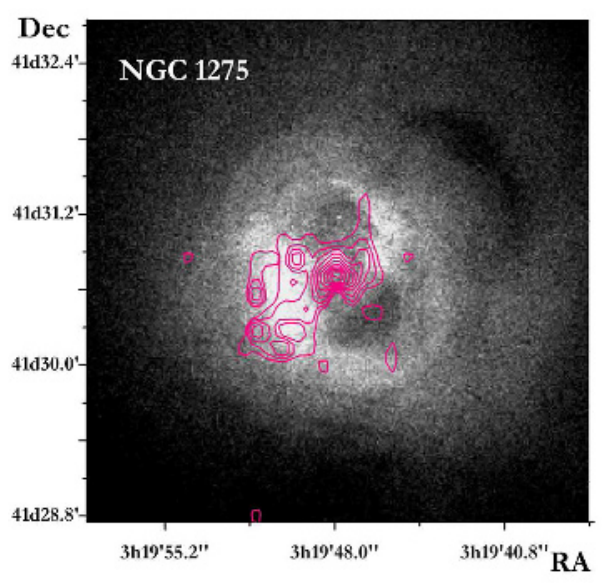

Figure 4. Chandra X-ray image [13] of NGC 1275 together with SHALON data. The contour lines show the TeV - structure by SHALON observations.

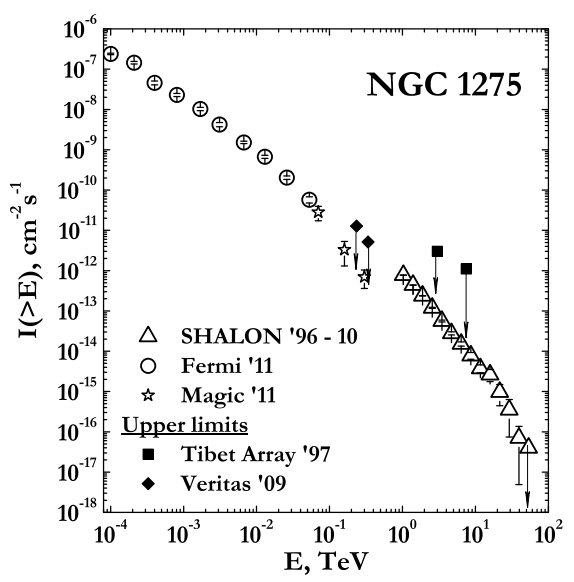

Figure 5. The TeV energy spectrum of NGC 1275 from SHALON, 15 year observations in comparison with other experiments: Fermi LAT [9], Veritas [10], Tibet Array [11], MAGIC [12];

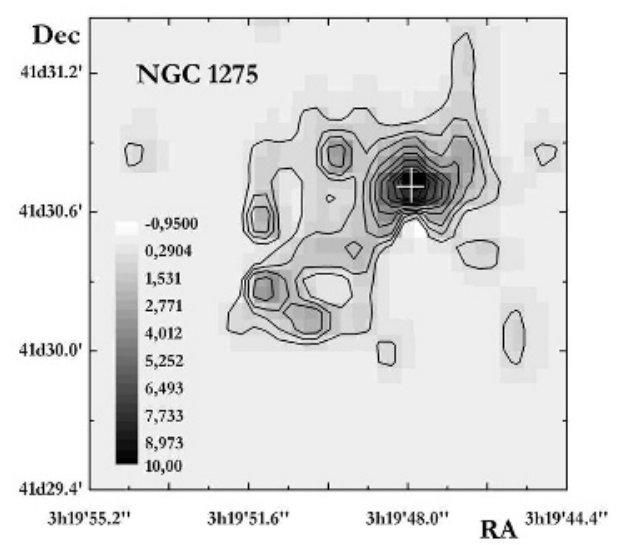

Figure 6. The NGC 1275 image at energy range of $>800 \mathrm{GeV}$ by SHALON.

(with redshift $\mathrm{z}=0.0179$ ); its image is shown in Figs. 4, 6. The maxima of the TeV gamma-ray, X-ray [13] and radio emission coincide with the active nucleus of NGC 1275. 


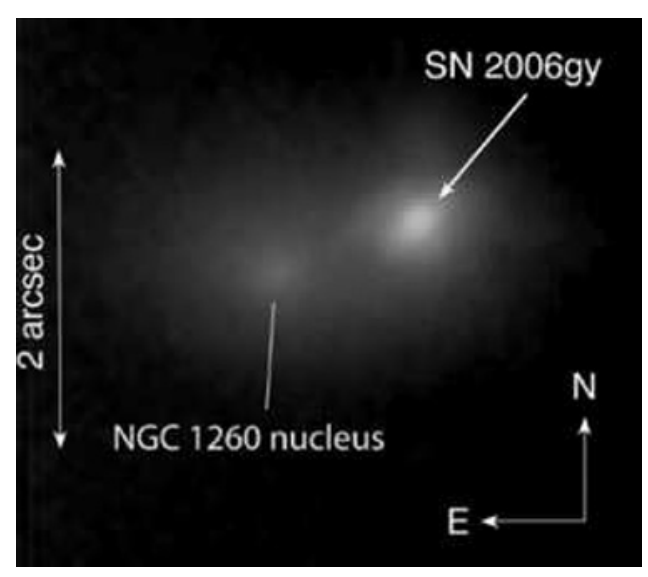

Figure 7. The image of SN2006 gy (Chandra) and the nucleus of NGC 1260 at three wavebands: J band(1.25 $\mu \mathrm{m}), \mathrm{H}$ band (1.65 $\mu \mathrm{m})$, and $\mathrm{Ks}$ band $(2.2 \mu \mathrm{m})[14]$.
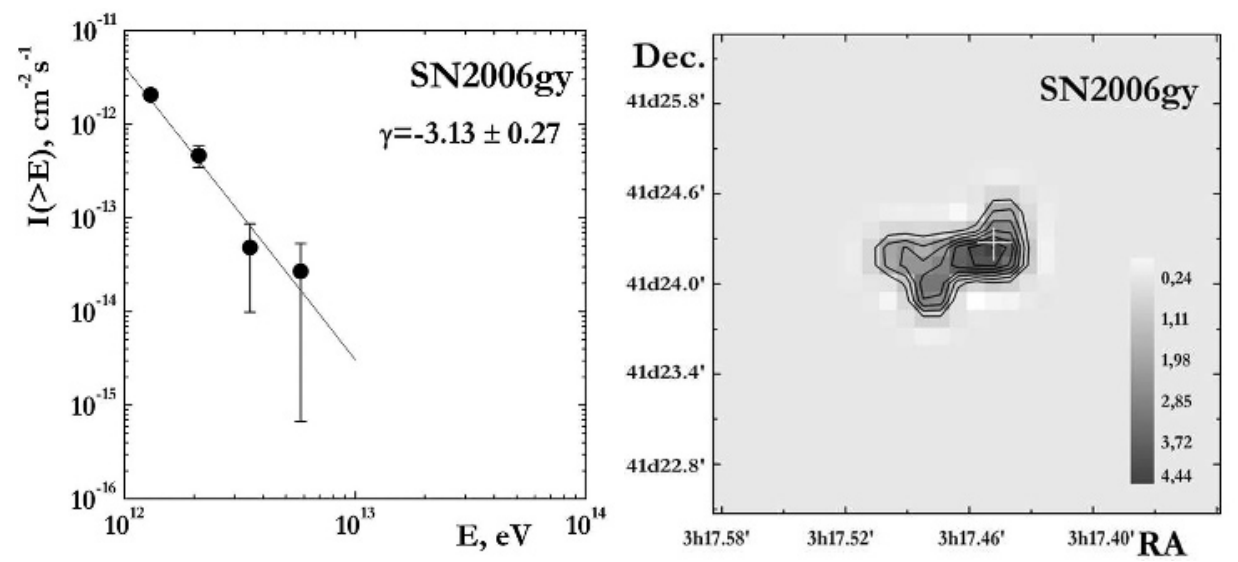

Figure 8. The SN2006gy by SHALON: left: the $\gamma$-quantum integral spectrum; right: The SN2006gy image at TeV energy range.

In contrast, the $\mathrm{X}$-ray and $\mathrm{TeV}$ emission disappears almost completely in the vicinity of the radio lobes. The correlation $\mathrm{TeV}$ with $\mathrm{X}$-ray emitting regions was found. The gamma-ray emission from the position of NGC 1275 was detected above $800 \mathrm{GeV}$ with a statistical significance [7] of $29.1 \sigma$ (for 263.4 hours). The integral $\gamma$-ray flux for this source is found to be $(0.78 \pm 0.05) \times 10^{-12} \mathrm{~cm}^{-2} \mathrm{~s}^{-1}$ at energies of $>800 \mathrm{GeV}$. The energy spectrum of NGC 1275 at 0.8 to $40 \mathrm{TeV}$ can be approximated by the power law $F\left(>E_{O}\right) \propto E^{k_{\gamma}}$, with $k_{\gamma}=-2.24 \pm 0.09$. The variations of the gamma-ray flux on the year scale are no more than $20 \%$ of average value.

\section{SN2006 gy}

The flux increase was detected from the region NGC 1275 in autumn 2006. The detailed analysis of $\gamma$-shower direction turned out the detection of metagalactic object. This object was identified with the supernova SN2006 gy (see Fig. 7)and [15] that is about 10 minutes away from NGC 1275.

Observations had been done in cloudless nights of moonless periods of 2006 September, October, November, December and then during the winter of 2007 . No flux increase was found in September observations. In the flare, observed on October 22, the flux increased 6 times from the NGC 1275 average flux and stayed on this level all October moonless period. After the standard analysis, a excess corresponding to a $6.2 \sigma$ [7] was determined. The integral gamma-ray flux for SN 2006gy is found to be $(3.71 \pm 0.65) \times 10^{-12} \mathrm{~cm}^{-2} \mathrm{~s}^{-1}$ at energies of $>0.8 \mathrm{TeV}$. The energy spectrum of SN2006 gy at 0.8 to $7 \mathrm{TeV}$ can be approximated by the power law $F\left(>E_{O}\right) \propto E^{k_{\gamma}}$, with $k_{\gamma}=-3.13 \pm 0.27$ (Fig. 8, left). An image of gammaray emission from SN2006 gy by SHALON telescope is shown in Fig. 8, right. Follow-up observations on end of November showed that the flux of SN2006 gy had dropped to a flux level of about $(0.69 \pm 0.17) \times 10^{-12} \mathrm{~cm}^{-2} \mathrm{~s}^{-1}$ and was constant during the November, December period. The results of observation analysis of 2007 have no revealed $\mathrm{TeV}$ gamma-ray emission from region of SN 2006gy. So, the explosion of extragalactic supernova was observed at $\mathrm{TeV}$ energies for the first time with SHALON Cherenkov telescope.

No other significant variations of the $\gamma$-quantum flux during the observations of NGC1275 were found during all the period of observations. 


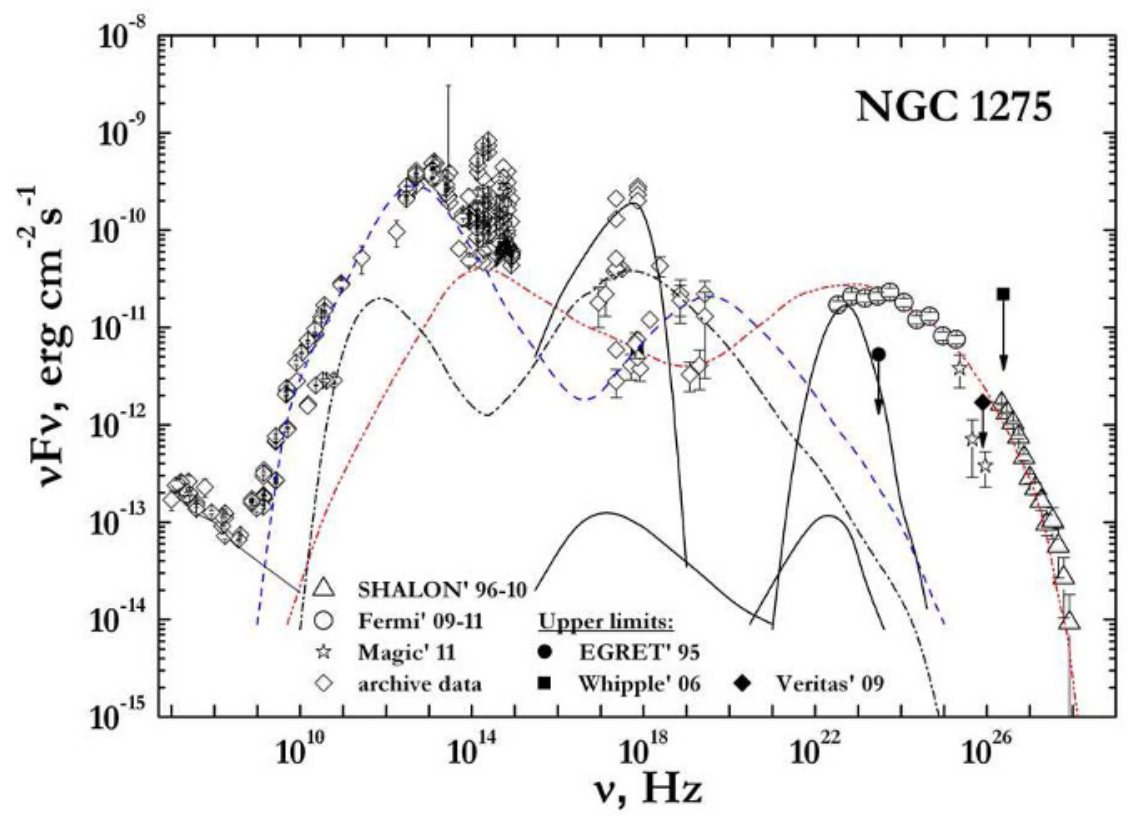

Figure 9. Overall spectral energy distribution of NGC 1275. The TeV energy spectrum of NGC 1275 from SHALON, 15 year observations in comparison with other experiments: Fermi LAT [9], MAGIC [12] and upper limits: EGRET [16], Whipple[17], Veritas [10] and models [18].

\section{VHE observations of NGC 1275}

Experimental limits on the very high energy $\gamma$-ray emission from the Perseus cluster and its central galaxy NGC1275 have been obtained by the other experiments [9-12, 16, 17]. The Seyfert galaxy NGC 1275 has been also observed with the Tibet Array [11] (5 TeV) and then with Veritas [10] telescope at energies about $300 \mathrm{GeV}$ at 2009 and MAGIC [12] at 2011. The radio-galaxy NGC 1275 has been recently detected by Fermi [9] as a source of high-energy $\gamma$-rays with an average flux and powerlaw photon index of $F(>100 \mathrm{MeV})=(2.31 \pm 0.13) \times$ $10^{-7} \mathrm{~cm}^{-2} \mathrm{~s}^{-1}$ and $\gamma=-2.13 \pm 0.02$, respectively. The recent detection by the Fermi LAT of high-energy $\gamma$-rays from the radio galaxy NGC1275 makes the observation of the very high energy $(E>100 \mathrm{GeV})$ part of its broadband spectrum particularly interesting.

The overall spectral energy distribution of NGC1275 from the low energies to the $\mathrm{TeV}$ energies is presented (Fig. 9), where the spectrum of NGC 1275 from SHALON 15 year observations is also shown. Lines on Figure 9 show the fit to the multi-frequency spectral energy distribution of NGC 1275 obtained in the CM model [18] with the composition of three Synchrotron Self-Compton (SSC) components, generated by three separated plasma blobs blasted away from the NGC 1275 nucleus.

In this model, the second, smaller blob produces a SSC emission which fits well Fermi data at MeV-GeV energies and SHALON data at energies $800 \mathrm{GeV}$ to $40 \mathrm{TeV}$ (Fig. 9, red curve); it is also consistent with MAGIC and WHIPPLE upper limit at $\mathrm{GeV}-\mathrm{TeV}$ energies.

\section{Conclusion}

The Perseus cluster as a many other galaxy clusters is expected to be a source of gamma-ray emission due to various emission mechanisms.

The long-term observations of NGC 1275 which is central galaxy of the Perseus Cluster have been carried out with SHALON telescope. In 1996 year NGC 1275 are detected by SHALON at TeV energies [1-5]. The results of fifteen-year observation of NGC 1275 at energies from $800 \mathrm{GeV}$ to $40 \mathrm{TeV}$ are presented. A CM model [18] of gamma-ray emission of the Perseus cluster atmosphere and NGC 1275 is considered to describe the spectral properties of NGC 1275 in whole energy range. The Fermi LAT data at high energies and very high energy data from SHALON observations are well fitted in this model with three components [18], in which the most energetic and smaller blob produces a SSC emission observed at gamma-ray energies from $\mathrm{MeV}$ to $\mathrm{TeV}$ (Fig. 9, red curve), whereas leptonic and hadronic models [18] of diffuse gamma-ray emission from the Perseus cluster (Fig. 9, black solid curves) predict more than 4 times lower $\gamma$-ray flux than it was detected and can't describe the spectrum observed at the high and very high energies.

\section{References}

[1] V. G. Sinitsyna, Proc. of Towards a Major Cherenkov Detector-V ed. O.C. de Jager, South Africa, 136 (1997)

[2] V. G. Sinitsyna et al., 16th European Cosmic Ray Symposium, ed. J.Medina, Spain, 367 (1998)

[3] V. G. Sinitsyna, AIP (Conf. Proc.) 515, 293 (2000) 
[4] V. G. Sinitsyna et al., Nucl. Phys. (Proc. Suppl.) 75A, 352 (1999); 97, 215 (2001); 122, 247, 409 (2003); 151, 108 (2006); 175-176, 463 (2008); 196, 442 (2009)

[5] V. G. Sinitsyna, Rad. Phys. and Chem. 75, 880 (2006)

[6] J. Kataoka et al., Astrophys. J. 740, 29 (2011)

[7] T.-P. Li and Y.-Q. Ma, Astrophys. J. 272, 317 (1983)

[8] B. R. McNamara, R. W. O’Connell, C. L. Sarazin, Astrophys. J. 112, 91 (1996)

[9] A. A. Abdo et al., Astrophys. J. 699, 31 (2009)

[10] V. A. Acciari et al., Astrophys. J. 706, L275 (2009)

[11] M. Amenomori, in Proc. 26th ICRC, Salt Lake City 3, 418 (1999)
[12] J. Alekśic et al., Astron.\&Astrophys. 539, L2 (2012)

[13] A. C. Fabian, J. S. Sanders et al., Mon. Not. R. Astron. Soc. 318, L65 (2000)

[14] N. Smith et al. Astrophys. J. 666, 1116 (2007)

[15] E. O. Ofek, P. B. Cameron et. al., arXiv:astro$p h / 0612408 v 1$

[16] D. J. Thompson et al., Astrophys. J. Suppl. 10, 209, 259 (1995)

[17] J. S. Perkins et al., Astrophys. J. 644, 148 (2006)

[18] S. Colafrancesco et al., Astron.\&Astrophys. 519, A82 (2010) 